\title{
CHALLENGES ON THE ELABORATION OF EDUCATIONAL KITS FOR THE CONSTRUCTION OF ROLLED STEEL CONNECTIONS USING 3D PRINTING
}

\author{
S. González-Rodrigo, L.A. Zurita-Diaz, G. García-López-de-la-Osa, \\ F. Magdalena-Layos, B. González-Rodrigo \\ Universidad Politécnica de Madrid (SPAIN)
}

\begin{abstract}
The visualization of the encounters between rolled steel pieces that concur in a connection is sometimes difficult to understand in the learning process of how to build this kind of structures. Previous experiences using structural models and kits have shown improvements on the understanding of the subject because students are able to reproduce in a practical way, using those physical models, the building solutions that they have learned theoretically. The limitations of these structures should be also taken into account. In which, in addition to the pieces preparation, it is need to weld them in order to strengthen the connections. This allows highlighting the priority of some elements among others, the necessity of continuity of some of them according to the local solicitation, the order of the welding and the need for cuts in some pieces for their connection. This work pretends to analyse the advantages of the use of models done by $3 \mathrm{D}$ printing in the motivation of the students in the learning experience.
\end{abstract}

There have been many difficulties in the planning, design and elaboration of the pieces to get a complete structural kit. To achieve a versatile model which allows reordering its pieces for reproducing different joints has required solving different problems. Starting with the choice of an accurate scale for the management, visualization and subsequent custody of the models in every case, as well as the selection of the most suitable profiles for the representation of multiple joints.

On the other hand, the choice of the material, the thickness and the filling of the pieces, the same with the management of printing times and the difficulties in the balance of the pieces have required a redesign of the prototype in order to minimize material quantities, printing lengths, defective pieces, etc. and to increase the performance in the elaboration of the proposed models. In the present research work, the difficulties encountered during the process of designing and elaborating an educational kit to support the learning of rolled steel connections are highlighted.

Keywords: STEM experiences, experiential learning, structural models, 3D models impression.

\section{INTRODUCTION}

The development of teaching traditionally based on master classes is in continuous adaptation to the new methodologies that emerged in the implementation of new requirements of the EHEA. The use of cooperative work and practical workshops are some of the tools used to complement the teaching activities [1,2]. The laboratories of structural models where destructive and non-destructive tests take place constitute an excellent complement to the theory taught in the classroom, allowing the students to assimilate knowledge through experimentation and transforming them into the protagonists of their learning process [3-5].

The reduced scale models allow the analysis and the understanding of how the geometric and dimensional characteristics of the material and the connection influence on the behaviour of the structures [6]. Likewise, it is possible to check how different constructive elements with structural function have different responses according to different load scenarios [7]. Therefore, it is possible to achieve a significant progress in the simulation of basic behaviours of the joints of the different structural elements explained in the theoretical lessons. In addition, attending to the different load hypothesis, design and structural connections exposed. Allowing checking visually the concepts of load transmission, strains, buckling instability and the variables on which they depend on.

Nowadays, tools available for our students as 3D printers, allow us to create models with a greater precision, which facilitates the isolation of a structural behaviour, object of analysis, from other variables of the execution of the models itself. 3D printing can obtain almost any geometry; however complex it may be [8].This versatility offers a great opportunity for teaching, allowing the creation of experimental 
material in order to improve the learning process on a workshop of structural models and keep this material for future lessons.

Through the creation of models and the experimenting with them, the concepts seen in the theoretical lessons related to stresses, structural systems, construction systems, materials, types of joints, etc. help the students to get and have a deeper approach to them. In addition, they can see complex concepts such as slenderness and reflect about the importance of the overall and partial stability of the structures and how execution decisions on site can significantly influence the final behaviour of the structure.

\section{METHODOLOGY}

That is why, as part of a project of educational innovation, at the Escuela Técnica Superior de Edificación and at the Escuela de Ingeniería Civil of the Universidad Politécnica of Madrid, structural kits have been made in the 3D printing. Those are scale models of different rolled steel pieces that allow the construction of structural standard structural joints in the classroom. The main objective of elaboration is to reinforcement the specific competences, such as the behaviour of constructive elements, how the structures resist and the background of the constructive systems with different materials.

When carrying out the 3D printing of the pieces that make the kits for the construction of structural joints of rolled steel, different difficulties have occurred that have made it troublesome and have delayed the realization of them.

\subsection{Devices and Materials Used}

In order to develop the printing Abax PRi5 3D printers have been used. The consumable filament used is made of Lactic Polyacid, commonly known as PLA with a thickness in diameter of $1.75 \mathrm{~mm}$ from the brand "Smart Materials 3D" in silver colour (Figures 1, 2, 3 and 4).
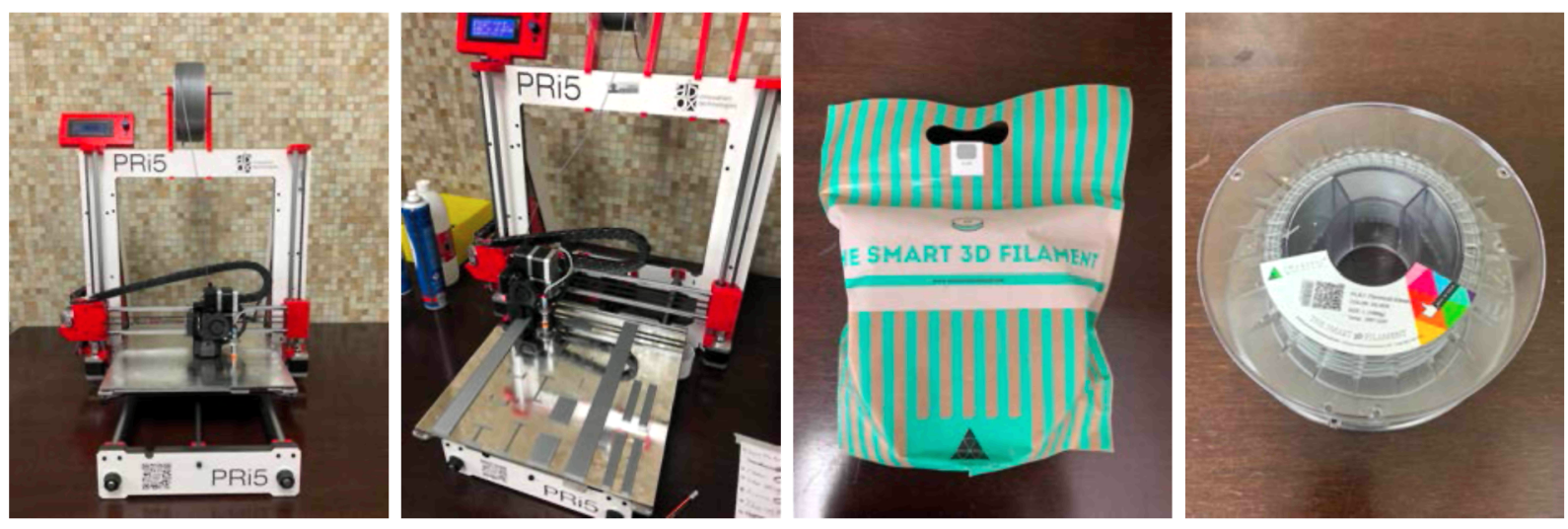

Figures 1-4. 3D Printer and consumable filament used in the manufacture of the kits.

The decision about the pieces that should be designed and subsequently printed is based on the idea of getting the largest number of joint constructive solutions in a frame using the least amount of pieces. In that way, modelling a girder profile and a pillar profile, with the help of some auxiliary parts, it is possible to make different encounters between them.

A 3D CAD system has been used to design both the virtual model of the pieces and the possible joints between them (Figures 5 and 6 ). 

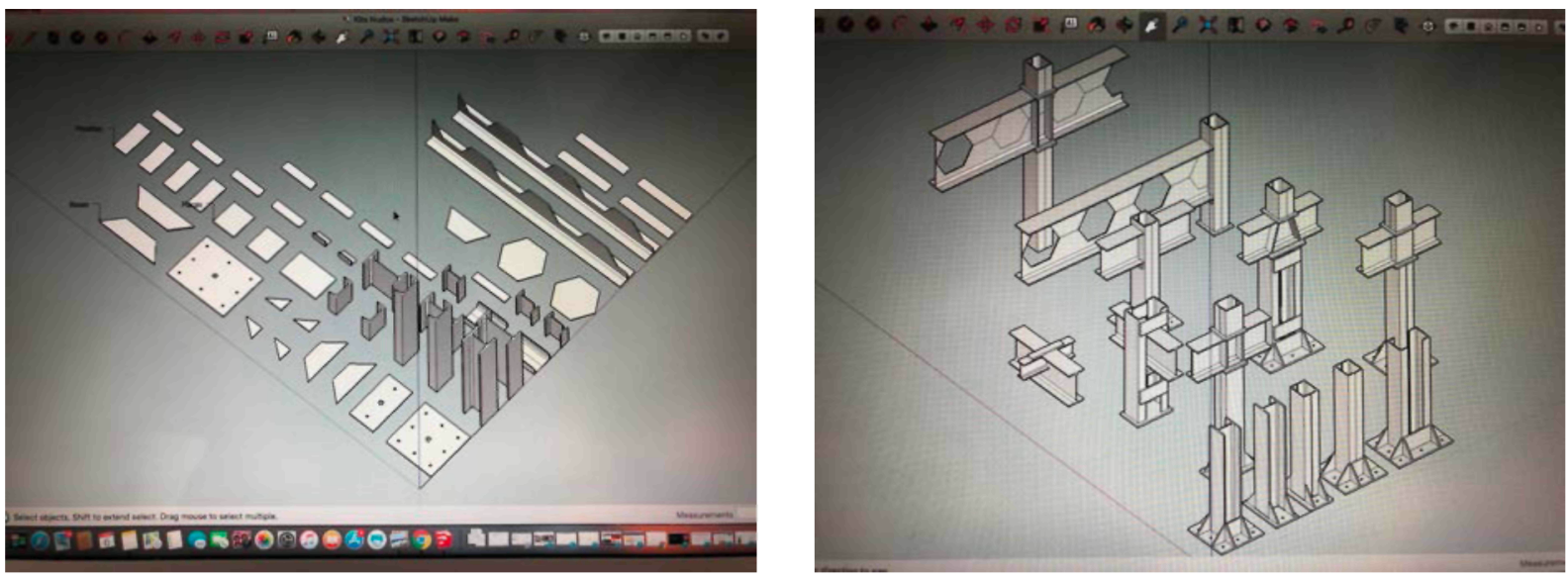

Figures 5,6. Virtual model of the pieces and possible joints between them.

Once the digital models of the pieces have been made, the joints studied, the set possibilities visualized and the pieces placed for printing, the print parameters (perimeter, padding, etc.) are determined by transforming the files to the software used for the printing (Ultimaker Cura) (Figure 7).

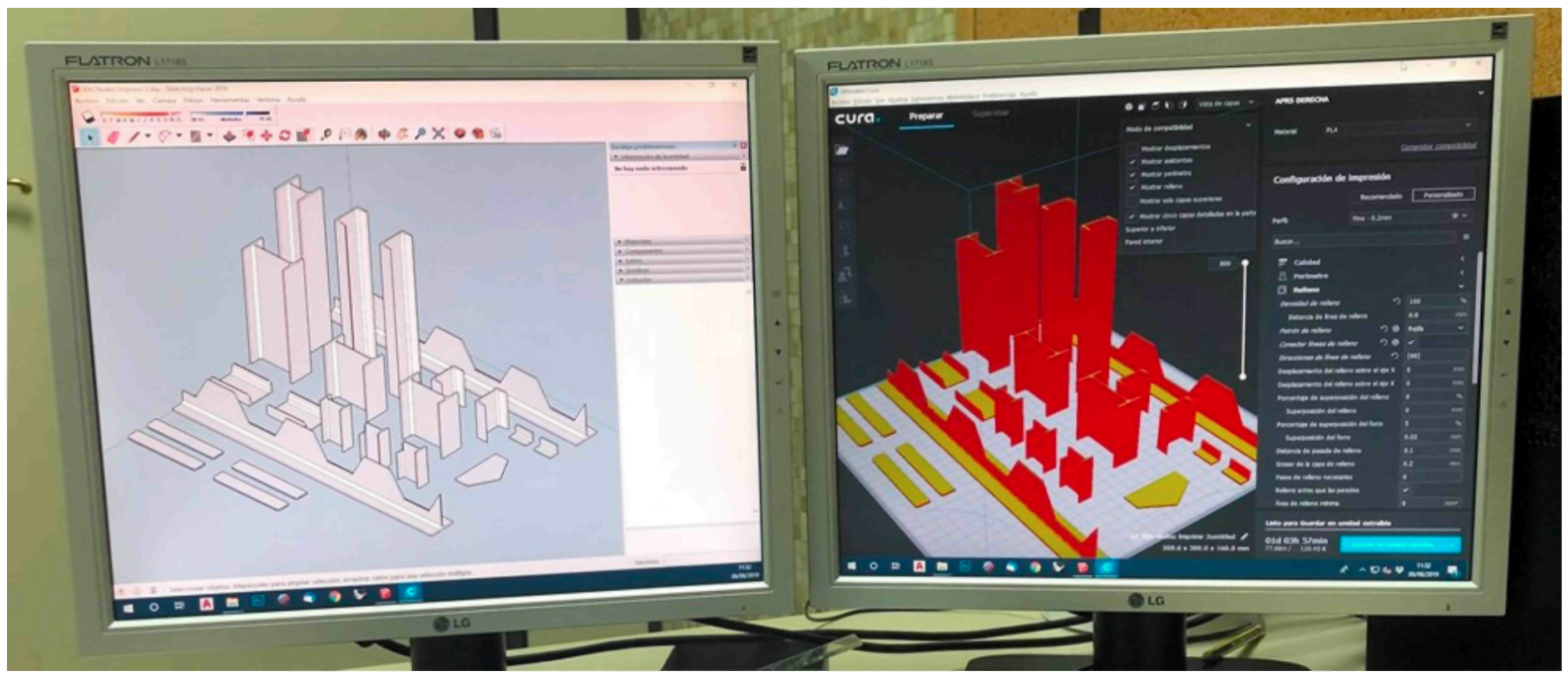

Figure 7. Transformation of the $3 D$ CAD model into the software for printing

The values that must be used according to the 3D printers characteristics are a thickness of the layers deposited in the printing of the models of $0.2 \mathrm{~mm}$ high with a width of the nozzle $0.4 \mathrm{~mm}$. The speed of $40 \mathrm{~mm} /$ second is fixed during all the process.

\subsection{Difficulties during the process of making the kits}

As mentioned above, numerous unexpected situations during the printing process of the structural kits have constitute a considerable delay, mainly due to the time required by the 3D printer to complete each piece, since, as we have seen, the deposit with PLA filament is made by $0.2 \mathrm{~mm}$ thick layers. Due to this situation, printing a complete kit should take approximately 50 hours. In addition, the limitations produced by the size of the printer bases used in the project $(30 \times 30 \mathrm{~cm})$, conditioned the use of two simultaneous printers or two consecutive prints on the same equipment to obtain a complete kit. However, the difficulties made this far from real.

The first problem encountered is related precisely to the long period required in each printing, which forces the entire set to be discarded if printing is interrupted for any reason. Therefore, the possibility of printing the larger pieces individually and the smaller ones grouped in batches is considered.

On the other hand, being scale models of rolled steel profiles and due to the slenderness of both the flange and the web, at the beginning it was considered necessary to print a fill that helped to support them. After several tests, and found that the advantages that in principle had been associated with this 
filling were not such and that the unnecessary consumption of material and the slowdown of the processes, advise against the incorporation of the filling into the print (Figure 8).

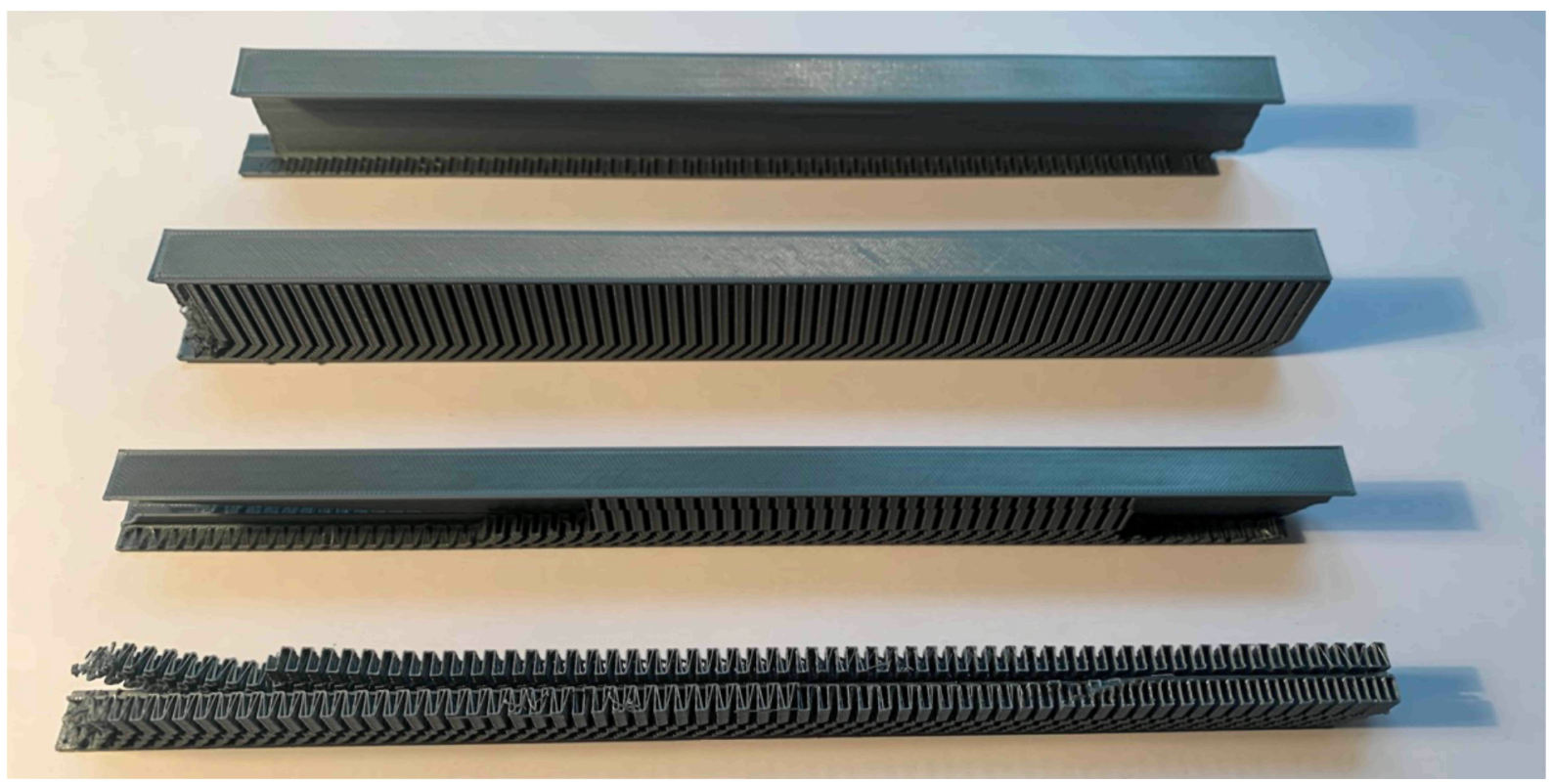

Figure 8. Fill considered at the beginning

Several causes have produce the failures during the printing. Overall, the most common have been the displacement of the printing on an axis that has ended the verticality of the piece, and the interruption of the thread exit by the mouthpiece that has left the piece unfinished. The first cause has been attributed to the pulleys worn out of one of the printers and the excessive movement in one direction, possibly caused by a decalibration due to the inertia of the piece (Figures 9 and 10). The second cause is mainly due to the jams that occur in the engines that ejects the thread (Figures 10, 11, 12 and 13), among others.
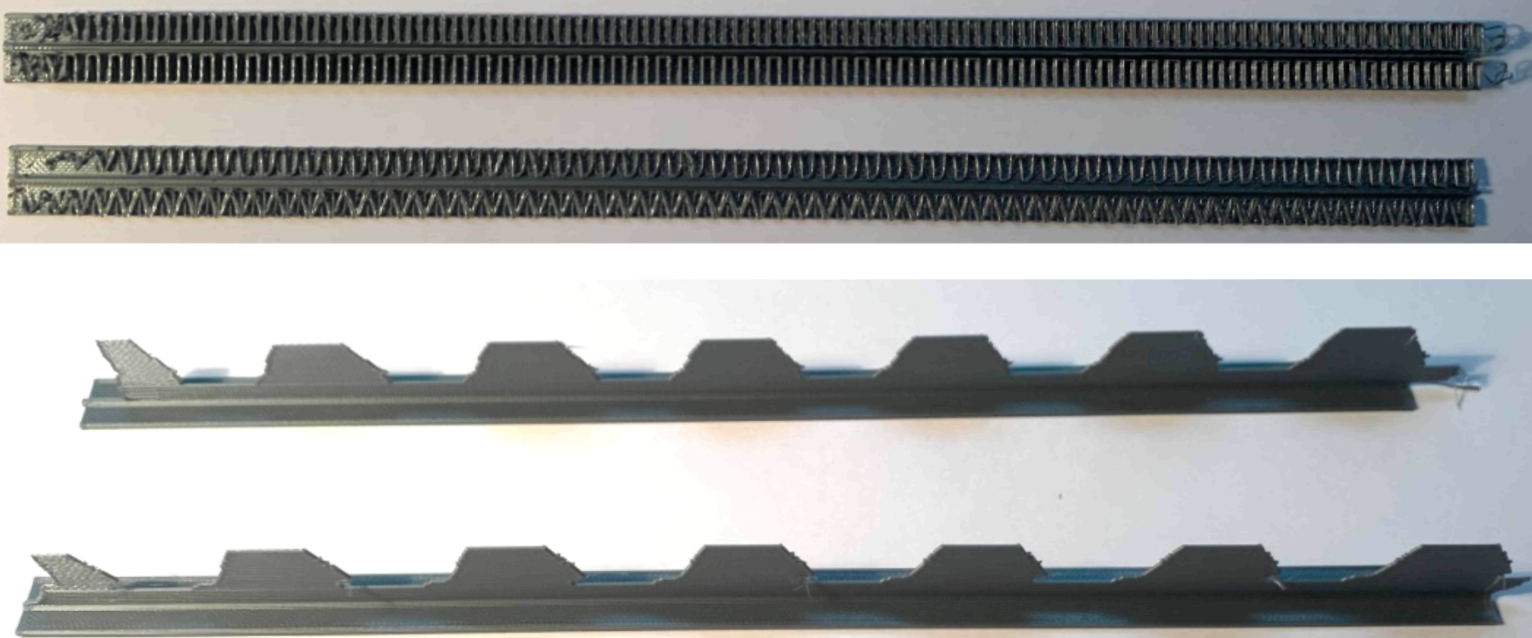

Figures 9, 10. Failures caused by worn out pulleys. 

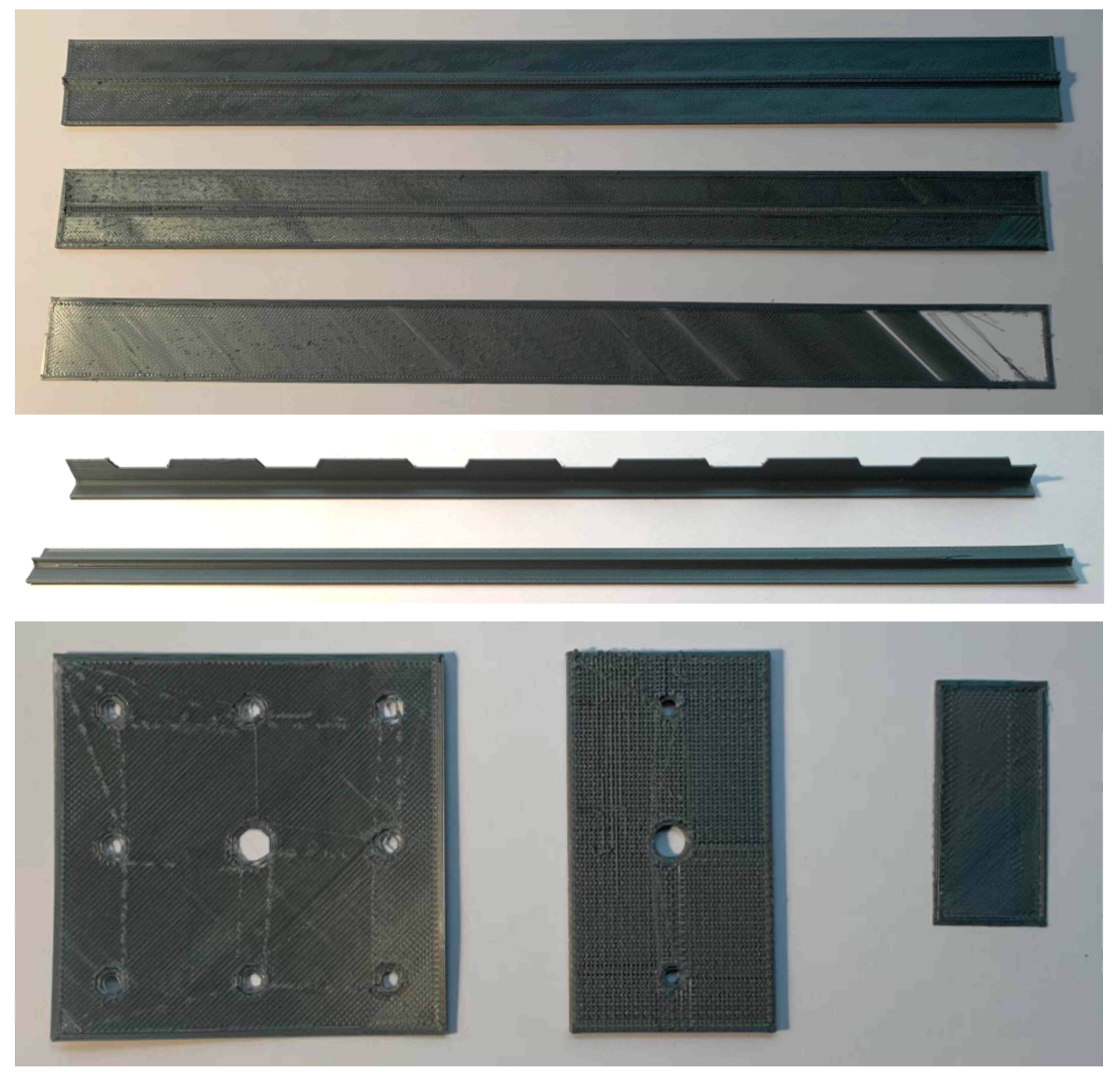

Figures 11-13. Failures originated by the total interruption of the filament output due to clogging or engine failure.

Sometimes the interruption of the thread is due to the fact that it remains attached to the nozzle and interrupts the deposit on the piece (Figures 14 and 15).
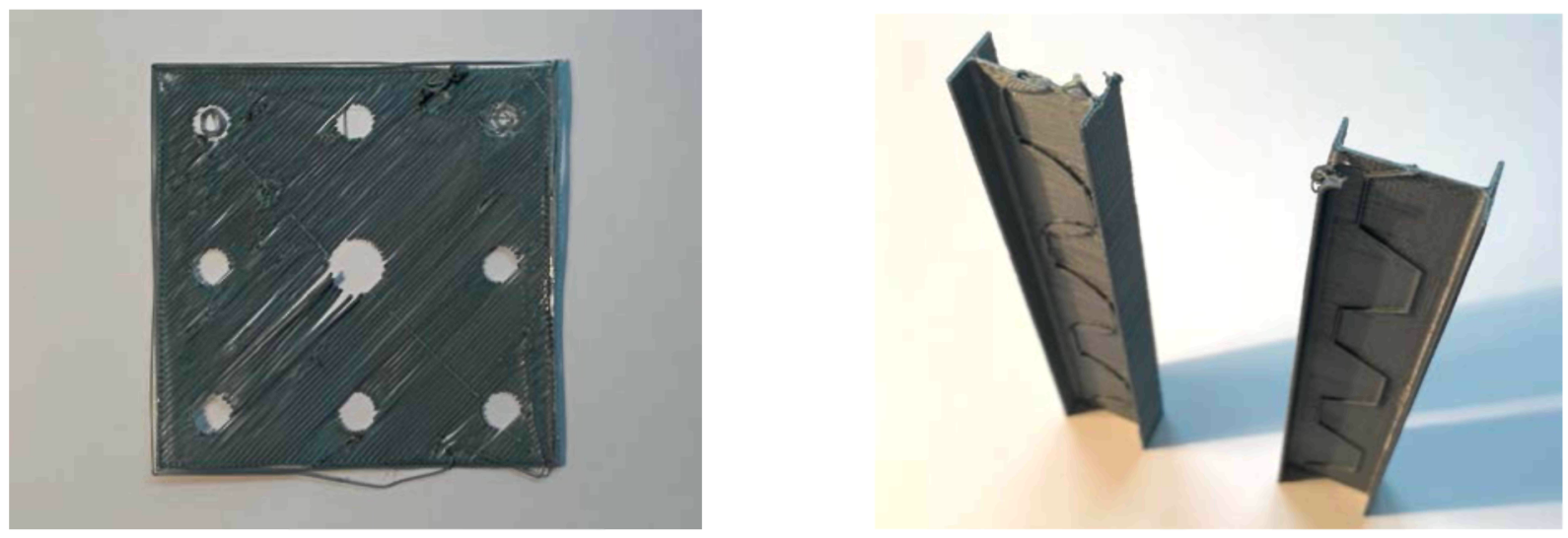

Figures 14, 15. Total interruption in the filament outlet due to thread stuck in the nozzle. 
Motor jams or failures can also cause partial interruptions in filament exit "Fig. 16". Another less common failure has been the poor adherence of the pieces to the base, which has caused displacements or separations of the pieces. For this reason, it has been necessary to find the balance between a clean final surface of the piece and an enough rough surface of the printing base, being necessary to spray the base with lacquer or adhesive before printing "Fig.17".
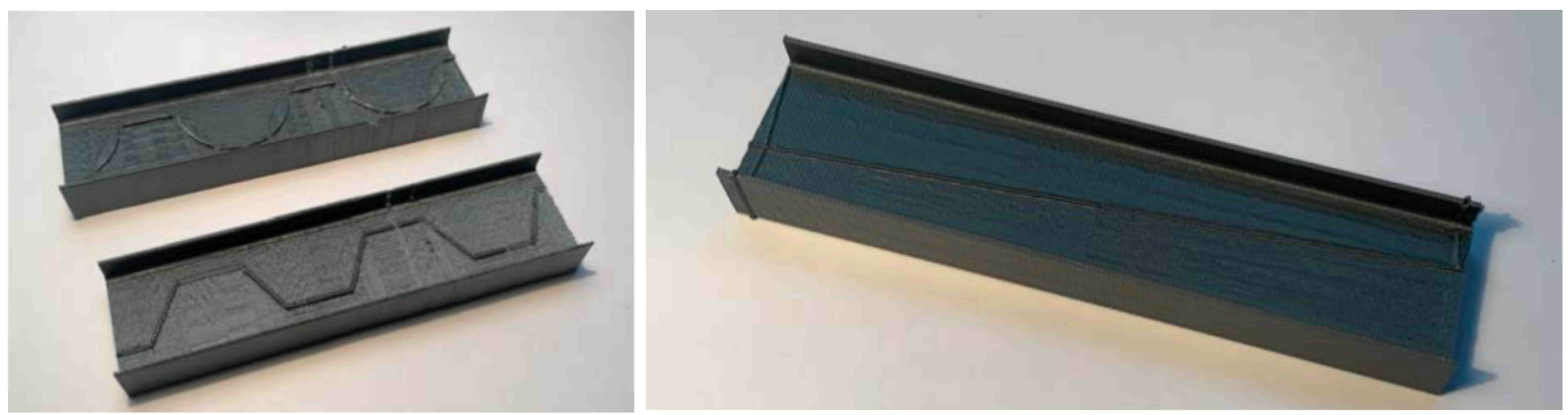

Figures 16,17. Partial interruption in filament output due to clogging or engine failure. Poor adhesion to the base.

On the other hand, the unexpected unevenness of the base with respect to the height of the nozzle has impede some impressions. It has also happened that the printed piece does not stick to the contour of the virtual model, which causes the pieces not to fit correctly in the place designed in the model. Modifications have been made (Figures 18 and 19).
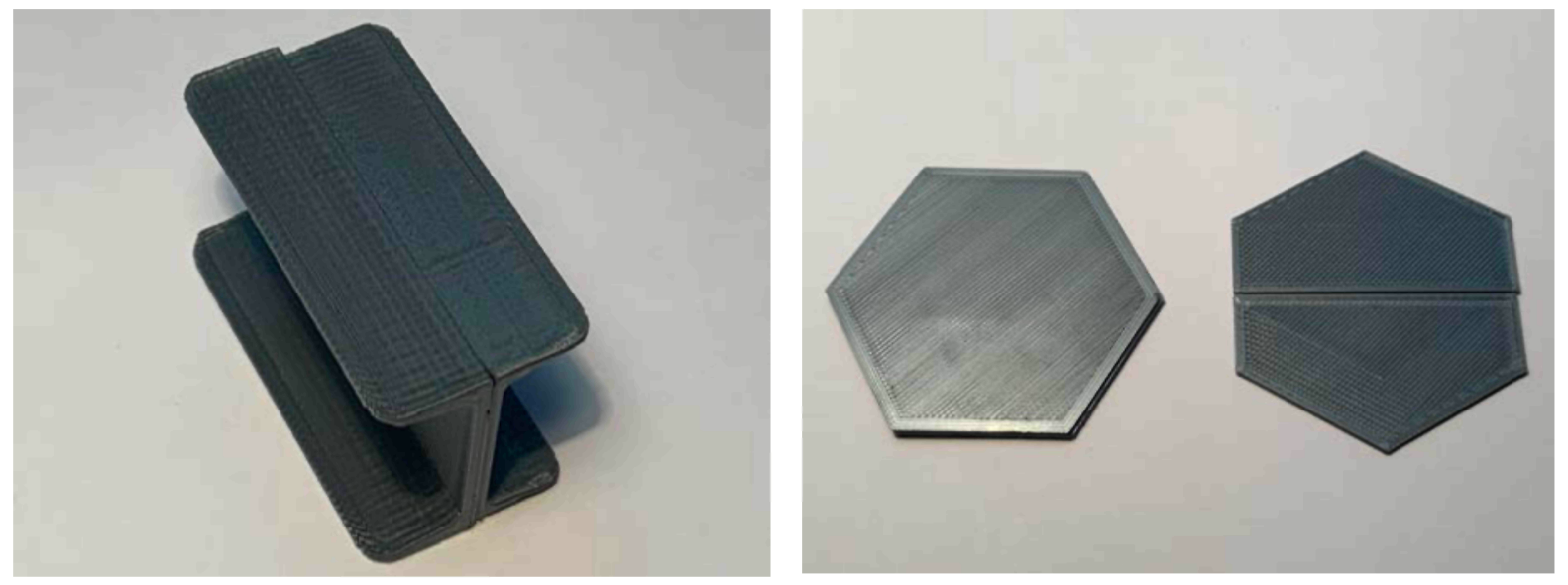

Figures 18,19. Modifications because the print does not stick to the edge.

It has also happened on one occasion that the coil that supplies the printing thread has run out of material, and also that there was a knot in the filament that has imped the stopped the supply.

During the deposit of the material, it happens that the nozzle can lose the calibration and disturbing the position of the deposit of the material, obtaining irregular geometry figures (Figure 20).

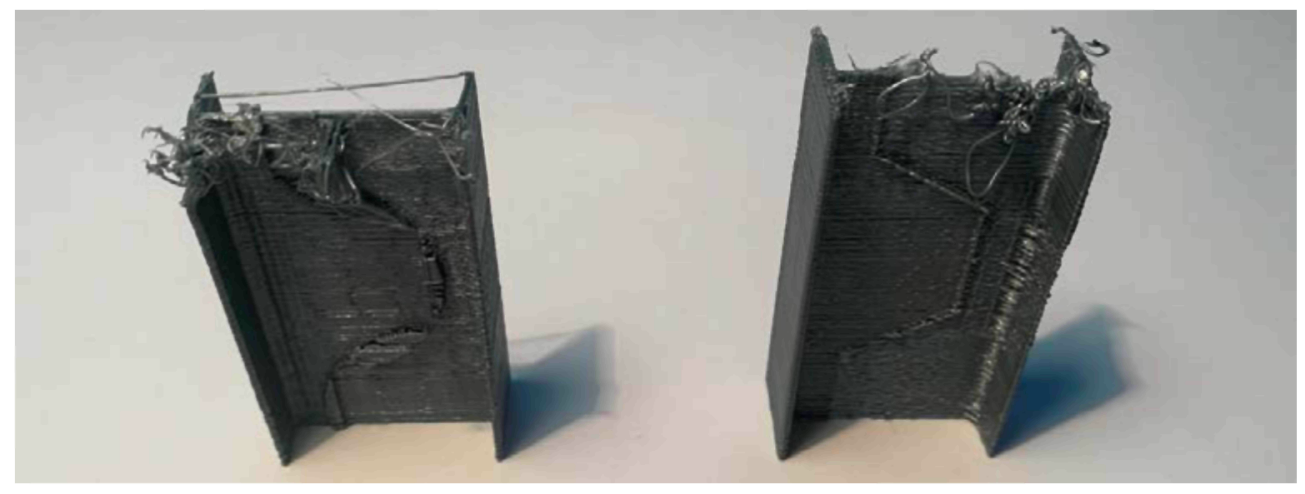

Figure 20. Decalibration of the nozzle: Due to its movement mechanism. 
The local accumulation of heat during the printing process, due to the thermal contribution necessary for the fusion of the thread also produces defects in the final pieces caused by the sharp contractions during the cooling of the piece (Figure 21)

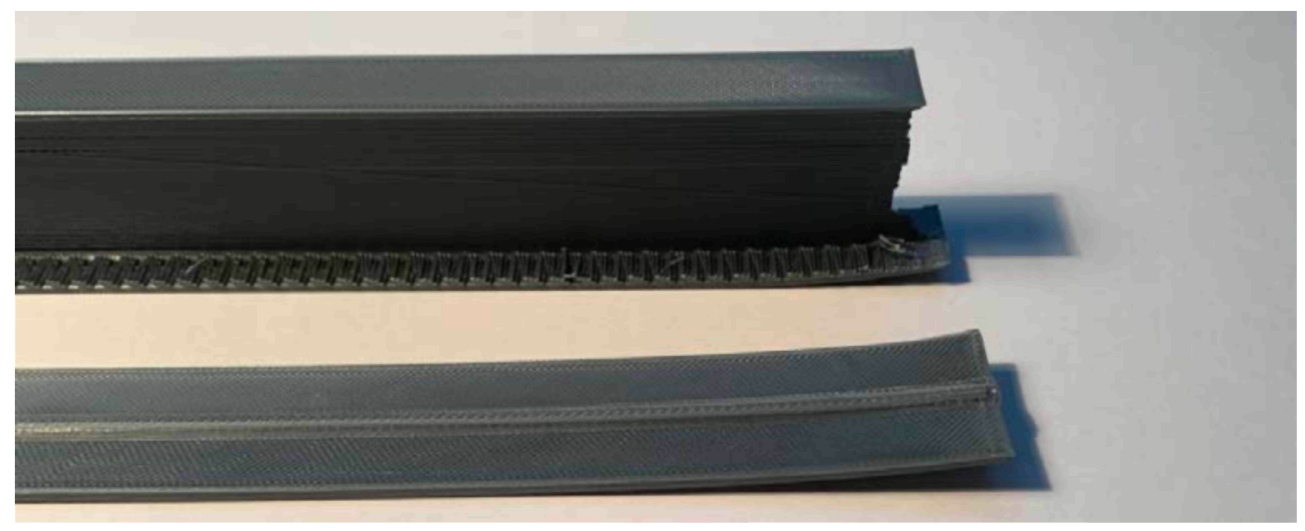

Figure 21. Lifting of one end of the piece due to contraction during cooling.

It is also important to take into account the fill patterns used by the printing software itself for the pieces. It may happen that some pieces have a texture or surface drawing, which is not as expected or as it is supposed to be (Figure 22)

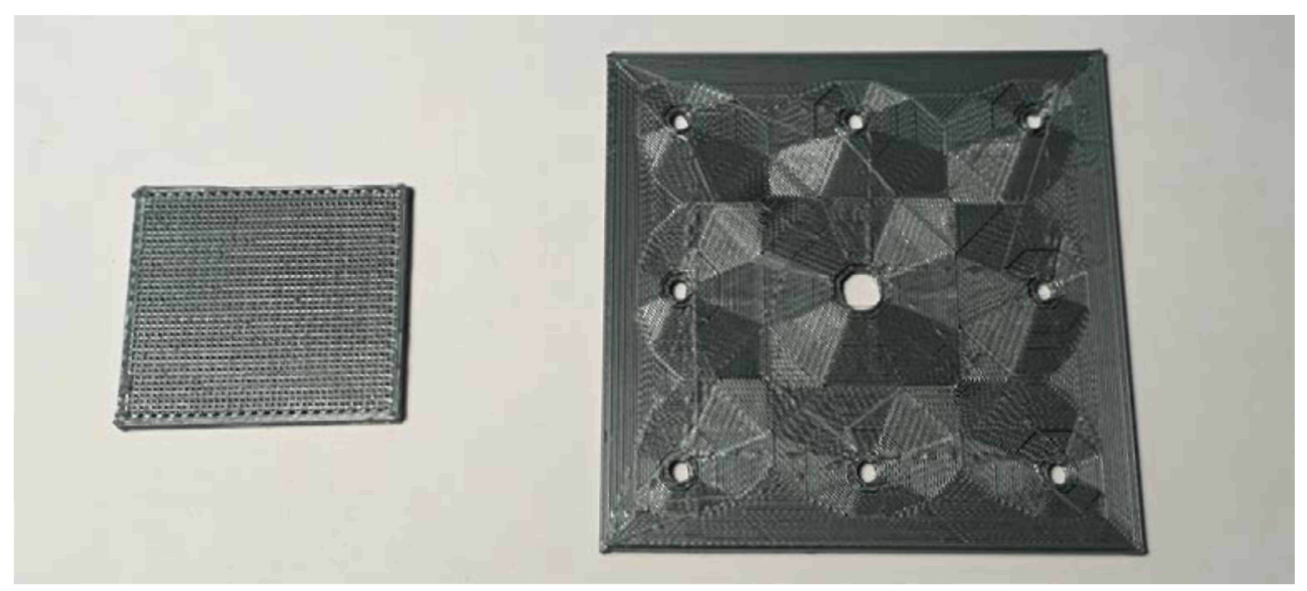

Figure 22. Unexpected surface texturing due to uncontrolled fill pattern.

Precisely a greater thickness has been used to highlight geometries, cuts, weld seams, etc. in steel pieces or encounters to facilitate the visual reading of the intended detail (Figure 23)

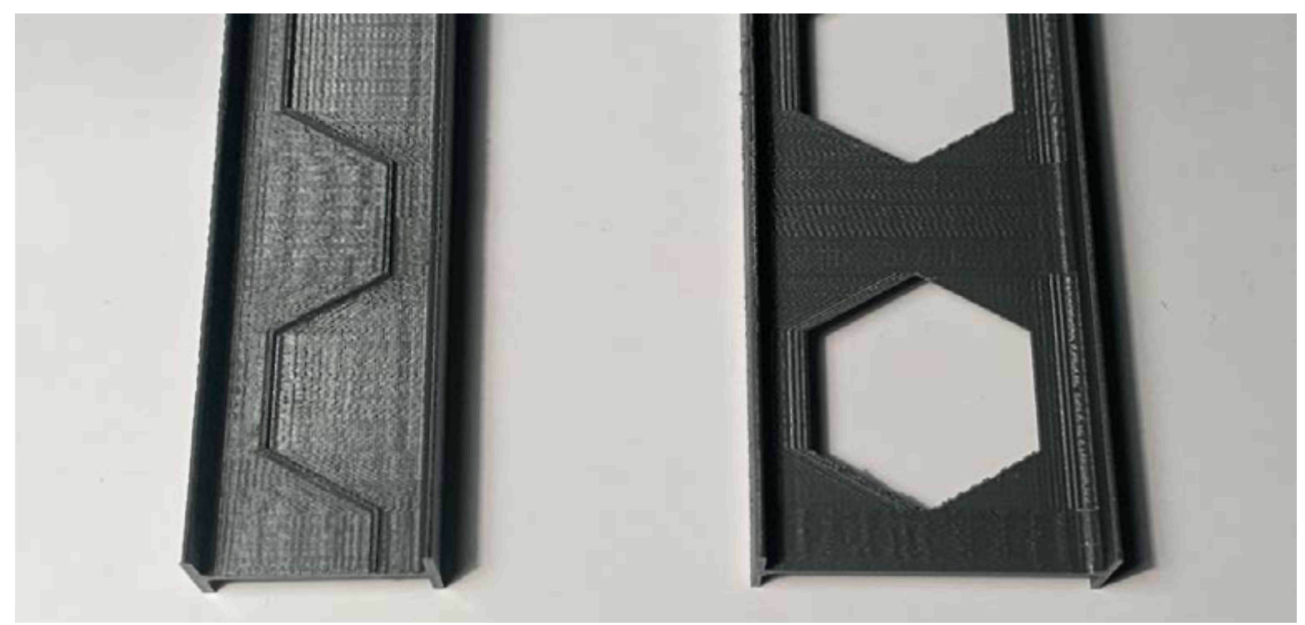

Figure 23. Higher thickness used to highlight cutting lines and weld seams. 
Another important factor to consider is the thickness of the walls of the elements to be made. Occasionally, it has been necessary to modify the thicknesses since some pieces printed, got broken in the direction of the deposit of the thread, at the shortest impact (Figure 24)

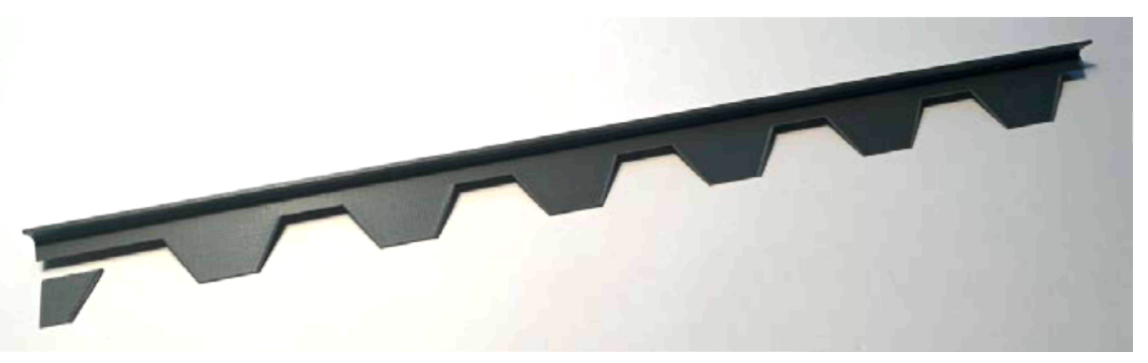

Figure 24. Breakage in the direction of the thread deposit due to an impact.

\section{CONCLUSIONS}

During the printing process, different aspects to improve have been observed, such as the reduction of wall lines because the pieces are solid, the establishment of the two-way filling for a better durability and the replacement of the circular figures with pentagonal ones for better adhesion of the walls with the filling.

Finally, once the pieces have been printed, it has been observed that joining pieces that should fit perfectly, the printer has not stuck to the contour; they are a few tenths of a millimetre larger indeed. That has made us make dimensional modifications of the virtual models, and reprint the pieces in a way the connection is correct.

Likewise, for some auxiliary pieces, it has been considered appropriate to reduce their thickness to make them see visual accurate and to make them resemble more closely the thicknesses of plates used in the building works for these joints.

\section{REFERENCES}

[1] P.C. Wankat and F.S. Oreovicz. "Teaching Engineering. New York", McGraw-Hill. 1993.

[2] M. Prince. "Does Active Learning Work? A Review of the Research". Journal of Engineering Education, 93(3), 223-231. 2004

[3] S. González-Rodrigo, B. González-Rodrigo F. Magdalena Layos, G. García-López de la Osa, "Experiential Learning through Scale Structural Models" Proceedings of ICERI2018 Conference, Seville, pp. 1928-1934, 2018. doi:10.21125/iceri.2018.1421

[4] S. González-Rodrigo, G. García-López de la Osa, F. Magdalena Layos, B. González-Rodrigo I, "Educational Kits in the Classroom: Learn by Testing" Proceedings of ICERI2018 Conference, Seville, pp. 1951-1954, 2018. doi: 10.21125/iceri.2018.1425

[5] N. Holmes and H. Mullen. "Using Model Building in Structural Engineering to Enhance Understanding of Construction Principles and Methods". Irish Journal of Academic Practice: Vol. 2(1). 2013. doi:10.21427/D7SX45.

[6] T. Ji. and A.J. Bell. "Seeing and Touching Structural Concepts in Class Teaching". Proceedings of the Conference on Civil Engineering Education in the21st Century, Southampton, UK, 26-28, 2000.

[7] M. Vrontissi. "The Physical Model in Structural Studies within Architecture Education: Paradigms of an Analytic Rationale?" Proceedings of the International Association for Shell and Spatial Structures (IASS), Amsterdam, 2015.

[8] S. Moyano Sanz and M. Valiente López "Printed thermoplastic modular piece, P.T.M.P". Building \& Management, vol. 2(1), pp. $12-16,2018$. 